\title{
Effect of the Use of WeChat to Disseminate Preoperative Health Education to Parents of Children with Restrictive Ventricular Septal Defects: A Prospective Randomized Controlled Study
}

\author{
Qi-Liang Zhang, ${ }^{1}$ Ning Xu, ${ }^{1,2}$ Shu-Ting Huang, ${ }^{1,2}$ Qiang Chen, ${ }^{1,2}$ Hua $\mathrm{CaO}^{1}$ \\ ${ }^{1}$ Department of Cardiac Surgery, Fujian Maternity and Child Health Hospital, Affiliated Hospital of Fujian Medical University, \\ Fuzhou, China; ${ }^{2}$ Department of Cardiovascular Surgery, Union Hospital, Fujian Medical University, Fuzhou, China
}

\section{ABSTRACT}

Background: This study explored the clinical effect of implementing WeChat-based preoperative health education for parents of children with restrictive ventricular septal defect.

Methods: A prospective randomized controlled study was conducted in a provincial hospital on the southeast coast of China. Participants were randomly divided into an intervention (WeChat) group $(\mathrm{n}=35)$ and a control group (leaflet) group $(n=35)$. The study explored the parents' knowledge of preoperative care, the follow-up rate, and complications of children with restrictive ventricular septal defect.

Results: The score of preoperative care knowledge of the WeChat group $(66.9 \pm 3.5)$ was significantly higher than that of the leaflet group $(47.3 \pm 6.3)(P=.006)$. Compared with the score of care knowledge at the first visit, the score of the WeChat group at the preoperation was significantly higher $(P$ $<.05$ ), whereas the increase in the score of the leaflet group was not significant. The rate of lost follow-up in the WeChat group $(0 \%)$ was significantly lower than in the leaflet group $(16.7 \%)(P=.02)$. The complications in the leaflet group were significantly higher than in the WeChat group.

Conclusions: Using WeChat to provide preoperative health education to parents of children with restrictive ventricular septal defect can effectively enhance the parents' knowledge of preoperative care and reduce the occurrence of complications and loss to follow-up.

\section{BACKGROUND}

A ventricular septal defect is one of the most common congenital heart diseases, and surgery is the main method to treat it [Anderson 1986; Hoffman 2002; Xing 2010]. Children with restrictive ventricular deficiencies who have no symptoms and low pulmonary arterial pressure are generally recommended to undergo surgical treatment after the age of 1 year.

Received Sepember 11, 2020; accepted October 14, 2020.

Correspondence: Hua Cao, Department of Cardiac Surgery, Fujian Maternity and Child Health Hospital, Affiliated Hospital of Fujian Medical University, Fuzhou,350001, China (e-mail: caobua0791@163.com).
Because of the left-to-right shunt of the heart, these children have poorer physiques and growth than healthy children, and it is more difficult to take care of them at home. Therefore, many parents feel stressed or anxious about the children's preoperative home care and have difficulty handling unexpected incidents [British Cardiac Society Working Party 2002; Wei 2015]. Studies have indicated that good preoperative care is associated with optimal surgical outcomes [Berg 2013]. Before the operation, we should provide sufficient health knowledge support for parents so that children can obtain better preoperative care. However, the current research has been focused on postoperative care and treatment, and the difficulty of home care before surgery is often ignored, especially for pediatric patients.

In recent years, many different social media software programs have been widely used in health management and education about diseases such as diabetes, hypertension, and coronary heart disease to improve clinical effects and reduce anxiety in patients and their families [Gabarron 2016; Xu 2015]. Smartphones are widely used around the world, especially in China [Hoedebecke 2017]. Mobile apps are becoming increasingly popular for health education, especially among busy people, who may not have time to visit medical institutions [Mihalko 2015]. In addition, the WeChat platform has been shown to disseminate health education more effectively than traditional information transmission methods in terms of shortening time consumption, reducing economic costs, improving treatment compliance, reducing complications, increasing follow-up rates, and improving patients' conditions [Lyu 2006]. However, there are few reports of the implementation of WeChat-based preoperative health education for parents of children with restrictive ventricular septal defects. This study aimed to evaluate the effectiveness of the implementation of WeChat-based preoperative health education for parents of children with restrictive ventricular septal defects.

\section{METHODS}

This study was approved by the ethics committee of Fujian Maternity and Child Health Hospital and strictly adhered to the tenets of the Declaration of Helsinki. In addition, all patients' guardians signed an informed consent form before 
the operation; code of ethics approval for scientific research project: no. 2020KY039. The datasets used and analyzed during the current study are available from the corresponding author on reasonable request.

\section{Research Design}

This was a prospective randomized controlled study conducted at a provincial hospital on the southeast coast of China. The clinical and family data of 70 patients with restrictive ventricular septal defects who were diagnosed and treated at our hospital from June 2017 to June 2018 were collected. Parents of all children completed a caregiver knowledge questionnaire at the first visit and before the operation. The general characteristics of all children and parents are shown in Table 1; differences between the 2 groups were not statistically significant. Differences in the scores of the parents' caregiver knowledge questionnaires at the first visit were not statistically significant. These results indicated that the 2 groups were homogeneous and comparable. Inclusion criteria were as follows: (1) children were diagnosed with a restrictive ventricular septal defect immediately after birth; (2) children were to receive selective surgery after 1 year of age; (3) parents were the primary caregivers; and (4) parents had smartphones and were able to use WeChat correctly. Exclusion criteria were as follows: (1) comorbid moderate or severe pulmonary hypertension; (2) need for surgical treatment before 1 year of age; (3) comorbid congenital heart disease; or (4) parents refused to participate in the study or follow-up plan.

\section{Calculation of Study Sample Size}

Based on the results from the pre-experiment and assuming that the $\alpha$ value was set at 0.05 with a power of 0.90 , the required number of participants was calculated to be 32 in each group. Assuming a $10 \%$ missing rate, the total sample size was set as 70 ( 35 per group).

\section{Intervention Methods}

In the WeChat group, the parents were provided health education and care guidance via the WeChat platform on their child's first visit, were guided on how to join the WeChat platform, and were taught to use WeChat functions correctly and skillfully. The health education content in the WeChat group mainly included 2 parts: the education module and the question-and-answer module. The education module included related knowledge on ventricular septal defect disease, preoperative care, family care and feeding, and the management of complications. Parents could view it and learn at any convenient time. For the question-and-answer module, 1 medical staff member of our team was on duty every day and online in the WeChat group at 18:00 to 22:00 to assist

Table 1. Demographic Characteristics of Patients and Their Parents in the 2 Groups*

\begin{tabular}{|c|c|c|c|}
\hline Age (d) & $18.0 \pm 8.4$ & $16.2 \pm 7.3$ & .203 \\
\hline Pulmonary pressure $(\mathrm{mmHg})$ & $22.5 \pm 4.9$ & $23.7 \pm 3.3$ & .329 \\
\hline Age of operation time (mo) & $16.5 \pm 2.9$ & $16.8 \pm 2.6$ & .506 \\
\hline$\leq 25$ & 3 & 4 & \\
\hline 26 to 30 & 10 & 9 & \\
\hline 31 to 35 & 12 & 11 & .953 \\
\hline 36 to 40 & 7 & 9 & \\
\hline$\geq 40$ & 3 & 2 & \\
\hline Junior college & 13 & 11 & \\
\hline Bachelor's degree or more & 7 & 6 & \\
\hline \multicolumn{4}{|l|}{ Living condition } \\
\hline Rural area & 23 & 24 & .799 \\
\hline City & 12 & 11 & \\
\hline Score of care knowledge at the first visit & $46.4 \pm 7.4$ & $45.3 \pm 6.9$ & .649 \\
\hline
\end{tabular}

*Data are mean \pm SD or $\mathrm{n}$. 
parents with their problems, provide reminders, supervise regular outpatient reviews, and remind parents of operation times. The medical staff also guided the family members in the WeChat group to communicate, discuss, and share their care experiences and actively encourage each other.

The parents in the control group obtained a leaflet at the first visit. The leaflet contained the same educational information as the intervention group and the time of re-examination and operation. The parents were also told to visit the hospital immediately in the event of an emergency.

\section{Data Acquisition}

The researchers divided eligible parents into the intervention group (WeChat group) and the control group (leaflet group) based on computer-generated random numbers. The researchers screened eligible parents for study and collected relevant data. The parents were told not to disclose their grouping or share material with other parents (Figure 1).

\section{Research Tool}

The Congenital Heart Disease Caregiver Knowledge Questionnaire, designed by Professor Y.F. Qiu of Mackay Medical College in Taiwan, was used to assess the level of parental care knowledge. The content validity of the questionnaire is 0.95 , the homogeneity reliability is 0.84 , and the test-retest reliability is 0.82 . The questionnaire has 18 items, starting from 0 , and each item is scored 0 to 4 . The items were forward-scored, except for items 11, 13, and 16, which were reverse-scored. "Totally disagree" was scored 1 point, "tend to disagree" was scored 2 points, "tend to agree" was scored 3 points, and "totally agree" was scored 4 points, for a total possible score of 72 points. Higher scores indicated better knowledge levels ( $<44$, low knowledge level; 44 to 58 , medium knowledge level; $>58$, high knowledge level).

\section{Statistical Analysis}

Continuous data were presented as the mean \pm standard deviation (SD) and range. The normal distribution test was performed on all continuous data, and they followed normal distribution. Clinical parameters between the 2 groups were compared with the independent samples $t$ test. The $\chi^{2}$ or

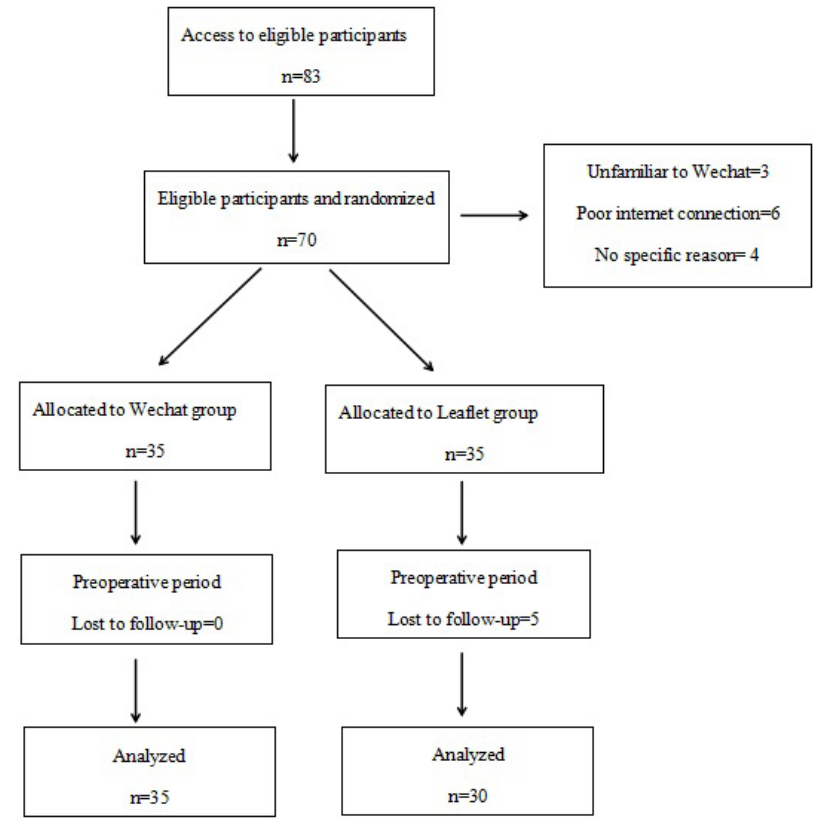

Figure 1. Details of the study population

Fisher's test was used to categorize the variables. A P value of $<.05$ was defined as significant.

\section{RESULTS}

The results of the knowledge test showed that the difference in the scores between the WeChat group $(46.4 \pm 7.4)$ and the leaflet group $(45.3 \pm 6.9)$ was not significant $(P=$ $.649)$ at the first visit. Preoperatively, the preoperative care knowledge score of the WeChat group $(66.9 \pm 3.5)$ was significantly higher than that of the leaflet group $(47.3 \pm 6.3)(P$ $=.006)$. Compared with the score of care knowledge at the first visit, the score of the WeChat group at the preoperative visit was significantly higher $(P<.05)$, whereas the increase in the score of the leaflet group at the preoperative visit was not significant (Table 2).

Table 2. Comparison of Parental Care Knowledge Scores, Loss to Follow-Up, and Complications Between the 2 Groups*

\begin{tabular}{|c|c|c|c|}
\hline Score of care knowledge before operation & $66.9 \pm 3.5$ & $47.3 \pm 6.3$ & $.006 \dagger$ \\
\hline \multicolumn{4}{|l|}{ Complications } \\
\hline Pneumonia & $5(14.3)$ & $13(16.7)$ & .009 \\
\hline Heart failure & 0 & 0 & \\
\hline
\end{tabular}

*Data are mean \pm SD or $n(\%)$.

$\dagger P<.05$ compared with care knowledge score at the first visit. 
All children in the WeChat group were followed up, but 5 children $(16.7 \%)$ in the leaflet group were lost to follow-up $(P=.02)$. Complications were mainly lung infection, delayed growth and development, and heart failure. The incidence of pneumonia and delayed growth and development in the WeChat group was significantly lower than that in the leaflet group $(P=.009$ and $P=.026$, respectively). No heart failure was observed in either group (Table 2).

\section{DISCUSSION}

With the advancement of medical technology, the early detection rate of congenital heart disease has significantly increased, and more ventricular septal defects are found before and after delivery [Wiechec 2015]. Because no obvious symptoms or pulmonary hypertension, or only mild pulmonary hypertension, occur in children with restrictive septal defects, surgical treatment is recommended after 1 year of age [Zhu 2013]. Congenital heart disease is often more serious than other diseases, and there is a left-to-right shunt in ventricular septal defects. The growth and physical fitness of these children are often worse, so preoperative care is relatively more difficult [British Cardiac Society Working Party 2002; Wei 2015].

China is a developing country, and advanced medical care is mainly concentrated in large cities. The level of basic medical care is relatively backward, and knowledge about cardiac surgery is poor. The families of children with congenital heart disease mainly live in rural areas, and their education level is low. They know little about congenital heart disease, and they cannot master this knowledge from a leaflet and through their outpatient experience. In this study, $62.9 \%$ of children lived in rural areas, and $81.4 \%$ of children's family members had an education level of college or below. The family members had little knowledge about congenital heart disease. Many parents were concerned about the home care of children before surgery. The caregivers felt stressed or anxious and had difficulty handling unexpected events. Therefore, it was very important to implement new strategies to guide parents in their preoperative care.

Mobile medical technology has been widely used as an educational tool in health care services [Hafiji 2012; Feng 2017]. In Europe, mobile apps have been used to promote health education for older people, maintain antiviral therapy, and reduce anxiety levels in HIV patients [Longinette 2017]. In the UK, mobile apps are used to encourage cancer survivors to actively participate in physical health programs [Puszkiewicz 2011]. In New Zealand, mobile apps are used for preoperative preparation before colonoscopy [Liu 2014]. All these studies supported the use of mobile apps as effective educational tools and proved that they can improve clinical outcomes. WeChat is a popular, convenient, and intuitive method of information exchange, and it integrates functions such as graphics, text, audio, and video to maximize information coverage [Kokol 2016].

In our study, WeChat was used in the preoperative care of children with restrictive ventricular septal defects. Through WeChat, parents can learn from education modules anytime and anywhere, according to their needs. When they have questions, they can consult medical staff through WeChat anytime and anywhere and obtain timely and effective answers and professional knowledge support. In this way, parents' knowledge of preoperative care can be effectively improved so that children can obtain better care before surgery. Because of the low level of education and the uneven distribution of medical information in China, most of the parents in the leaflet group could not effectively obtain knowledge from their leaflet or consult professional doctors in a timely manner. Therefore, the knowledge level of parents in the WeChat group was significantly higher than that of parents in the leaflet group, and the complications of lung infection and malnutrition in the WeChat group were significantly lower than those in the leaflet group.

All children in the WeChat group were followed up, but 5 children (16.7\%) in the leaflet group were lost to follow-up. The lost to follow-up rate of patients in the WeChat group was significantly lower than that of patients in the leaflet group $(P$ $=.02$ ). Through the WeChat group, medical staff can directly interact and communicate with the parents and can promptly and repeatedly remind them of the follow-up visit time, which can effectively help them remember to attend the follow-up visit. This communication method is not restricted by region or time; it is sustainable, low-cost, and very convenient; and it can effectively reduce the loss of contact between the family and the doctor. Parents in the leaflet group received only a leaflet with educational information and the date of follow-up after the first visit, and there was more than a year from the first visit to the operation. These parents were likely to ignore important conditions (because the child has no symptoms) and forget to go to the hospital for a follow-up visit.

\section{Limitations}

This study has some limitations. First, due to unstable internet support, especially in rural areas of China, many people cannot access WeChat; therefore, nearly $11 \%$ of qualifying parents were not recruited. Second, this was a singlecenter study.

\section{Conclusion}

WeChat-based health education is convenient, economi$\mathrm{cal}$, and interactive and can effectively implement various health education program. The use of WeChat to disseminate preoperative health education for parents of children with restrictive ventricular septal defects effectively enhances their knowledge of preoperative care and reduces the occurrence of complications and loss to follow-up.

\section{ACKNOWLEDGMENTS}

We gratefully acknowledge the contribution by the participating doctors: Xian-rong Yu, Ze-wei Lin, Wang-shen Dai, Ya-li Huang, Qiu-yu Chen, and Li-wen Wang.

\section{REFERENCES}

Anderson RH, Becker AE, Tynan M. Description of ventricular septal defects or how long is a piece of string? Int J Cardiol 1986;13:267-278. 
Berg K, Arestedt K, Kjellgren K. Post-operative recovery from the perspective of day surgery patients: A phenomenographic study. Int J Nurs Stud 2013;50:1630-1638.

British Cardiac Society Working Party. Grown-up congenital heart (GGCH) disease:current needs and provision of service for adolescents and adults with congenital heart disease in the UK. Heart 2002;88(suppl 1):11-14.

Feng S, Liang Z, Zhang R, Liao W, Chen Y, Fan Y, Li H. Effects of mobile phone WeChat services improve adherence to corticosteroid nasal spray treatment for chronic rhinosinusitis after functional endoscopic sinus surgery: A 3-month follow-up study. Eur Arch Otorhinolaryngol 2017;274:1477-1485.

Gabarron E, Wynn R. Use of social media for sexual health promotion: A scoping review. Glob Health Action 2016;9:321193.

Hafiji J, Salmon P, Hussain W. Patient satisfaction with post-operative telephone calls after Mohs micrographic surgery: A New Zealand and U.K. experience. Br J Dermatol 2012;167:570-574.

Hoedebecke K, Beaman L, Mugambi J, et al. Health care and social media: What patients really understand? F1000 Res 2017;6:118.

Hoffman JL, Kaplan S. The incidence of congenital Heart disease. Am Coll Cardiol 2002;39:1890-1900.

Kokol P, Blaun Voner H, Saranto K. Co-operation and co-funding networks in ehealth research. Stud Health Technol Inform 2016;225:950-951.

Liu X, Luo H, Zhang L, Leung FW, Liu Z, Wang X. Telephone-based reeducation on the day before colonoscopy improves the quality of bowel preparation and the polyp detection rate: A prospective, colonoscopistblinded, randomised, controlled study. Gut 2014;63:125-130.

Longinette E, Manoharan V, Ayoub H, Surkan PJ, El-Khatib Z. Utilization of social media and web forums by HIV patients: A cross-sectional study on adherence and reported anxiety. Prev Med Rep 2017;6:137-143.

Lyu KX, Zhao J, Wang B, et al. Smartphone application WeChat for clinical follow-up of discharged patients with head and neck tumors: A randomized controlled trial. Chin Med J (Engl) 2006;129:2816-2823.

Mihalko MC. We need to talk: The impact on care when electronic communication replaces verbal communication between clinicians. J Pediatr Nurs 2015;30:626-627.

Puszkiewicz P, Roberts AL, Smith L, Wardle J, Fisher A. Assessment of cancer survivors' experiences of using publicly available physical activity mobile application. JMIR Cancer 2011;2:1-19.

Wei H, Roscigno CI, Hanson CC, Swanson KM. Families of children with congenital heart disease: A literature review. Heart Lung 2015;44:494-511.

Wiechec M, Knafel A, Nocun A. Prenatal detection of congenital heart defects at the 11-week to 13-week scan using a simple color Doppler protocol including the 4-chamber and 3-vessel and trachea views. J Ultrasound Med 2015;34:585-594.

Xing Q, Pan S, An Q, Zhang Z, Li J, Li F, Wu Q, Zhuang Z. Minimally invasive perventricular device closure of perimembranous ventricular septaldefect without cardiopulmonary bypass:multicenter experience and mid-term follow-up. J Thorac Cardiovasc Surg 2010;139:1409-1415.

Xu J. Application of WeChat Public Platform in the Health Education of the Hospital. Health Educ China 2015;31:86-87.

Zhu D, Gan C, Li X, An Q, Luo S, Tang H, Feng Y, Lin K. Perventricular device closure of perimembranous ventricular septal defect in pediatric patients: Technical and morphological considerations. Thorac Cardiovasc Surg 2013;61:300-306. 